\title{
A New Investigation of the 30 Doradus Region with the Australia Telescope Compact Array
}

\section{J. S. Lazendic ${ }^{1}$}

Centre for Astronomy, University of Western Sydney, Nepean, Australia

R. F. Haynes

Australia Telescope National Facility, CSIRO, Australia

J. R. Dickel

Astronomy Department, University of Illinois, Urbana, IL 61801, USA

P. A. Jones and G. L. White

Centre for Astronomy, University of Western Sydney, Nepean, Australia

M. Costa

Physics Department, University of Tasmania, Australia

\section{Introduction}

30 Doradus is a giant H II region in the Large Magellanic Cloud (Bode 1801). It is the nearest extragalactic giant $\mathrm{H}$ II region and the location of active star formation. The complex nature of this extended region provides an excellent opportunity to investigate the impact of massive stars on the structure of the interstellar medium. Specifically the presence of supernova remnants (SNRs) is expected to play an important role.

\section{A Search for Supernova Remnants within the Nebula}

A number of studies of 30 Doradus have resulted in the encouraging discovery of high velocity material, which could well have originated in SNRs (e.g., Meaburn 1984; Chu \& Kennicutt 1994). Since radio SNRs that "hide out" in star-forming regions would have a distinctly non-thermal radio spectrum, sensitive radio investigation at high resolution are needed to separate them from the surrounding thermal region.

Our recent image of the 30 Doradus region at a wavelength of $6 \mathrm{~cm}$ made with the Australia Telescope Compact Array is shown in Fig. 1. The half-power beamwidth is $1 . \prime 7$ and this is the most sensitive radio image ever made of the region. Our initial aim was to seek out non-thermal emission regions across the

\footnotetext{
${ }^{1}$ Astrophysics Department, School of Physics, University of Sydney, Sydney, Australia
} 


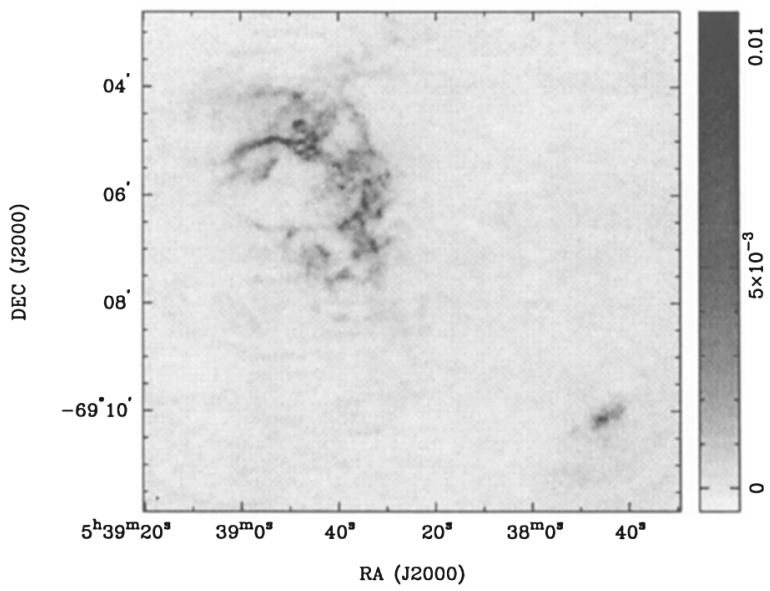

Figure 1. Radio continuum grayscale of 30 Doradus at $4.6 \mathrm{GHz}$ with a HPBW of 1 .'7. The peak brightness is $0.0108 \mathrm{Jy} \mathrm{beam}^{-1}$.

field of 30 Doradus or search for small-scale, non-thermal features within the nebula itself. A comparison of the maps at different radio frequencies showed that the emission of 30 Doradus does not vary significantly with frequency, which is consistent with a thermal spectrum of this object (Lazendic 1997).

\section{Hidden SNRs?}

To separate more clearly possible nonthermal components from thermal emission of the HII region, we compare our radio image with an $H \alpha$ image from the U Michigan/CTIO Emission Line Survey of the LMC conducted by R.C. Smith (1997). The radio and optical images show impressive similarities but also interesting differences. A scaled difference image between the radio image and the $H \alpha$ image revealed 5 unusual small-scale features that show a possible nonthermal nature. Further high-frequency high-resolution observations are underway to investigate the properties of these features.

The supernova remnant N157B (bottom right corner in Fig. 1) was also included in the study. This Crab-type object has a radio spectral index of -0.33 . Surprisingly, no polarised radio emission was detected from this SNR.

\section{References}

Bode, J. 1801, Algemeine Beschreibung und Nachweisung der Gestirne, (Berlin: Beym Verfasser)

Chu, Y.-H., \& Kennicut, R. C. 1994, ApJ, 425, 720

Lazendic, J. S. 1997, BSc Honours Thesis, U. Western Sydney Nepean

Meaburn, J. 1984, MNRAS, 211, 521

Smith, R. C. 1997, http://www.astro.lsa.umich.edu/ncels/mcsurvey.html 


\section{Discussion}

You-Hua Chu: Are the small-scale nonthermal radio source determined solely by comparison between radio and $\mathrm{H} \alpha$ images? How do you exclude the possibility of extinction effect?

Jones: Yes, the non-thermal radio features are determined by the comparison with the $\mathrm{H} \alpha$ image. We have not ruled out extinction yet. We need to compare the $\mathrm{H} \beta$ and $\mathrm{H} \alpha$ to determine extinction.

Ron Probst: A quick eyeball comparison of your differenced image with $\mathrm{my}_{2}$ image of 30 Dor appears to show that the compact $6 \mathrm{~cm}$ features all match compact $\mathrm{H}_{2}$ features - except one, seen in $\mathrm{Br} \gamma$, which is likely extinction related.

Jones: We are concerned about extinction and will compare $\mathrm{H} \beta$ and $\mathrm{H} \alpha$ images, and also correlate any radio features with other available data.

Rosa Williams: Is the southern feature seen near N157B real? If so, is it part of the outer shell, or is it pulsar-related?

Jones: The southern feature seen near N157B in the radio is part of the faint outer shell. There is no detected radio pulsar. The X-ray pulsar is in the middle of the radio SNR. 